\title{
An assessment of sleep quality and daytime sleepiness in hemodialysis patients: a cross-sectional study from Palestine
}

Ahmad M. Samara' ${ }^{1}$, Moutaz W. Sweileh ${ }^{1}$, Ali M. Omari ${ }^{1}$, Leen S. Omari ${ }^{1}$, Hazar H. Dagash ${ }^{1}$, Waleed M. Sweileh², Nehal Natour ${ }^{3}$ and Sa'ed H. Zyoud ${ }^{4,5,6^{*}}$ (D)

\begin{abstract}
Background: Patients on hemodialysis (HD) commonly suffer from poor sleep quality which in turn compromises their quality of life and well as their mortality risk according to many studies. In Palestine however, no single study had emphasized the association and prevalence of sleep quality and daytime sleepiness among hemodialysis patients (HDP). Therefore, we conducted this study to determine the relationship between regular HD and the situation regarding sleep quality and daytime sleepiness in HDP.

Methods: In this cross-sectional survey, we targeted HDP at one HD center in An-Najah National University Hospital. Sleep problems were assessed by both the Pittsburgh Sleep Quality Index (PSQI) and Epworth Sleepiness Scale (ESS), which are considered as standardized tools for the assessment of sleep quality and daytime sleepiness, respectively.

Results: Of the 192 dialysis patients who were interviewed, 167 were included in the final analysis of this study, with a response rate of $87 \%$. The mean age of the participants was $57.6 \pm 12.9$. Fifty-two percent were males, and an equal percentage of the participants were 60 years old or older. The median ESS of the participants was 7 [4-11] and $43.1 \%$ of the participants had 9 or more ESS score, indicating excessive daytime sleepiness. The median PSQI score was 8 [6-12] and $76.65 \%$ of the participants scored more than 5 points on the PSQI, indicating poor sleep quality. Neither the ESS score nor the PSQI score were significantly associated with any particular category of participants, based on their demographic or clinical characteristics.

Conclusions: In conclusion, we found that dialysis patients had poor sleep quality, evident by their high median PSQI score, though their excessive daytime sleepiness was less evident by their median ESS score in the high normal values. Additionally, we found a high prevalence of poor sleep quality among HDP, whereas excessive daytime sleepiness was less prevalent. Finally, no significant association was found between either of the two scores and the participants' demographic or clinical characteristics surveyed in this study.
\end{abstract}

Keywords: Haemodialysis, Sleep quality, Excessive daytime sleepiness, Palestine

\footnotetext{
* Correspondence: saedzyoud@yahoo.com; saedzyoud@najah.edu

${ }^{4}$ Poison Control and Drug Information Center (PCDIC), College of Medicine and Health Sciences, An-Najah National University, Nablus 44839, Palestine ${ }^{5}$ Department of Clinical and Community Pharmacy, College of Medicine and Health Sciences, An-Najah National University, Nablus 44839, Palestine Full list of author information is available at the end of the article
}

(C) The Author(s). 2019 Open Access This article is distributed under the terms of the Creative Commons Attribution 4.0 International License (http://creativecommons.org/licenses/by/4.0/), which permits unrestricted use, distribution, and reproduction in any medium, provided you give appropriate credit to the original author(s) and the source, provide a link to the Creative Commons license, and indicate if changes were made. The Creative Commons Public Domain Dedication waiver (http://creativecommons.org/publicdomain/zero/1.0/) applies to the data made available in this article, unless otherwise stated. 


\section{Background}

Chronic kidney disease (CKD) is defined as any structural or anatomical irreversible and progressive damage to the kidney (Naalweh et al. 2017). The last stage of CKD is known as End Stage Renal Disease (ESRD) (Kidney Disease: Improving Global Outcomes (KDIGO) CKD Work Group 2013). Patients who reach ESRD stage will need renal replacement therapy (RRT) either through kidney transplantation or maintenance dialysis (Abecassis et al. 2008; Kaza et al. 2015). In the West Bank in Palestine, a dramatic increase in the numbers of ESRD patients was observed over the last few years (Younis et al. 2015). In 2015, 1224 patients in the West Bank were documented to have ESRD and required hemodialysis (HD), revealing an increase of $>80 \%$ compared to 2011 (Ministry of Health, Palestinian Health Information Center 2016).

Hemodialysis patients' life quality can be impacted by many factors (Wang et al. 2016). Such factors include poor sleep quality, which was reported in many studies as a common problem among hemodialysis patients (HDP) and that it predicts their life quality in general, and their mortality risk (Wang et al. 2016). HDP, especially those with ESRD, may suffer physical, mental, and social consequences that can be the direct result of their ESRD, the therapy-related adverse effects on their quality of life, the lifestyle changes that come along with, or any number of these factors combined. In Palestine, HD centers were run by the Palestinian Ministry of Health, which suffers from under-staffing in all fields due to economic and political instabilities (World Health Organization 2012; Zyoud et al. 2014). Therefore, we expect that patients undergoing hemodialysis in Palestine may not receive adequate attention regarding sleep disorders by experts, to avoid any health complications. There are many tools available for the assessment of sleep quality and related sleep problems. Of these, two particular tests that are commonly and reliably used for that purpose: the Pittsburgh Sleep Quality Index (PSQI) and the Epworth Sleepiness Scale (ESS) which assess the quality of sleep and the excessive daytime sleepiness, respectively (Beaudreau et al. 2012).

Numerous studies regarding HDP were conducted and published in Palestine (Adas et al. 2014; Al-Ramahi et al. 2016; Zyoud et al. 2016a, b; Al-Jabi et al. 2018; Khatib et al. 2018; Omari et al. 2019); however, no single study emphasized the association and prevalence of sleep disorders among HDP. This study was conducted to determine the relationship between regular HD and the development of sleep disorders. The results of this study may help healthcare providers to, early on, implement strategies needed to improve HD outcomes and provide new data about the value of sleep disorders in HDP.

\section{Materials and methods Study design}

This study was developed and conducted as a cross-sectional, descriptive survey.

\section{Study setting}

The study took place in An-Najah National University Hospital's HD center in Nablus, which is considered the main HD center in the northern part of West Bank and provides its service to all the residents of Nablus district, estimated to be around 300,000 people.

\section{Study population, sampling procedure and sample size calculation}

As reported by the Palestinian Ministry of Health in their 2015 Health Annual Report, 210 dialysis patients were being treated at the An-Najah National University Hospital served by $33 \mathrm{HD}$ machines. The sample size for this study was determined using the Raosoft sample size calculator using a population size of $210 \mathrm{HDP}$, a $50 \%$ expected response of $50 \%$, a $5 \%$ error margin, and a $95 \%$ confidence interval. The calculated minimum number of participants needed in our sample was 134; however, a larger sample size of 192 HDP was selected to ensure more representative data. The study also used a convenience sample of HDP.

\section{Inclusion and exclusion criteria}

For inclusion criteria, we required that the participants be eighteen-years-old or older, fully aware, to have received HD for a minimum of 6 months, two sessions a week, each lasting $3 \mathrm{~h}$ or more, and have all the demographic, clinical, and biochemical data required for the study. In contrast, participants who could not hold a meaningful communication, for whatever physical, mental or other reason, were excluded from the final analysis.

\section{Data collection instrument}

A data collection instrument was used in this study, containing three sections. The demographic and social characteristics of the participants were the topic of the first section and included their age and sex, their marital status, residency, occupation, level of educational, monthly income, and their smoking status.

The second section addressed the participants' clinical status and contained the questions of how long they had had their diseases, how long the dialysis sessions were, how many dialysis session per week they underwent, what their body mass index was, and also noting the numbers of chronic diseases they had and the medications they used.

The last section included PSQI and ESS gradings (Buysse et al. 1989; Johns 1991). The PSQI test assesses sleep quality by exploring 7 areas which are the subjective quality of sleep, sleep latency, its duration, habitual efficiency, and disturbances, also the use of aiding sleep medication and daytime 
dysfunction (Buysse et al. 1989). This evaluation consists of two types of questions. The first is the open-ended type and there are 4 questions in this manner. The second type is answered on a 0 to 3 scale, indicating the frequency of troubled sleeping during the past week, wherein answering with 0 indicates having no trouble at all; with 1 , having trouble once; with 2, having trouble twice; and with 3 having trouble three times. The tested subject is considered to have poor sleep quality when his or her total score is 5 or higher. ESS, on the other hand, is used for the assessment of daytime sleepiness through items that address the question of how likely it is for a person to doze off, in various situations (Johns 1991). Its items are answered on a 0 to 3 scale to indicate the likelihood of dozing off, from 'not at all likely' to 'highly likely,' and reflected incrementally by the selected number. The subject's score is considered normal when his or her total sum is in the 0-10 range. We used previously translated and validated Arabic versions of the PSQI (Suleiman et al. 2010), and ESS (Ahmed et al. 2014) with permission. The questionnaire was administered to a pilot sample $(\mathrm{n}=15)$ to assess comprehensibility and to ensure that the questions were understood correctly. Internal consistency and reliability for ESS and PSQI were good and adequate, with Cronbach's $\alpha=0.741$ for ESS, and 0.726 for PSQI.

\section{Statistical analysis}

For data analysis, we used the IBM Statistical Package for the Social Sciences (SPSS), Version 21. In the results section, categorical variables were presented as frequencies, and both the percentages and continuous variables were either as means and standard deviations or as medians and inter-quartile ranges. Nonparametric MannWhitney test was used to compare two independent variables, and Kruskal-Wallis tests was used to compare multi-category variables (i.e. more than two independent variables), where appropriate. Kolmogorov-Smirnov test was used to assess normality. The significance level was set at $P<0.05$. Internal consistency reliability for ESS and PSQI were tested via Cronbach's alpha coefficient.

\section{Results}

Of the $192 \mathrm{HD}$ patients who were interviewed at AnNajah National University Hospital, 167 were included in the final analysis of this study, amounting to an $87 \%$ response rate. The socio-demographic, as well as the clinical characteristics of the participants, are presented, in full, in Table 1. The mean age of the participants was 57.6 with a standard deviation of 12.9. Fifty-two percent were males, and an equal percentage of the participants were 60 years old or older. Participants fell into normal, overweight and obese BMI categories by close percentages $(35,31$, and $31 \%$, respectively) and only a minority of $3 \%$ were underweight regarding their BMI. Thirtyseven percent of the participants received primary education as their highest level of education, which is the education category that contained the highest number of participants, followed by secondary, high, and graduation categories containing $19 \%$ each, and only $6 \%$ did not receive any formal education. Two thirds (67\%) of the participants came from a low-income household, 30\% from a moderate-income household and only $3 \%$ came from a high-income household. Based on their residency, 44\% were city dwellers, another $40 \%$ came from villages, and the rest $(16 \%)$ were living in refugee camps. The vast majority of the participants (94\%) were living with their families, and 78\% were married. Also, most participants (76\%) were unemployed at the time of the survey.

The details of the participants' dialysis history are also presented in Table 1. About 59\% of the participants had been on dialysis for less than 4 years, and the vast majority (95\%) of all participants were undergoing three dialysis sessions per week. Also, for $52 \%$ of them, the dialysis session duration was less than $4 \mathrm{~h}$. Most participants (90\%) did not have a previous transplantation history. About one-third of the participants had at least three total chronic co-morbidities, and $62 \%$ were on at least four chronically used medications.

The ESS scores, as well as the PSQI scores, for each of the participants' categories mentioned above, are listed in Table 2. The median ESS score of the participants was 7 (inter-quartiles: $4-11$ ) and $28.7 \%$ of the participants had a total ESS score of more than 10. The median PSQI score was 8 (inter-quartiles: 6-12) and as much as $76.65 \%$ of the participants scored more than 5 points on the PSQI. Neither the ESS scores nor the PSQI scores were significantly associated with any particular category of participants, based on their demographic or clinical characteristics (Table 2).

\section{Discussion}

In this study, we set out to investigate the quality of sleep and daytime sleepiness in HD patients. We found that HD patients in this study had poor sleep quality, evident by the high PSQI score (median 8, interquartiles 6-12) that significantly exceeded the cut-off point of 5 that is considered the upper limit of normal PSQI score, though their excessive daytime sleepiness was less evident, judged by the median ESS score of the participants that hovered in the high normal range, at 7 $(4-11)$. By viewing the data from another perspective and presenting the results in epidemiological terms, the prevalence of poor sleep quality (PSQI $>5$ ) among HDP in this study was $76.65 \%$, whereas the prevalence of excessive daytime sleepiness (ESS $>10$ ) among those patients was $28.7 \%$. Additionally, we found no significant association between either of these two scores and the participants' demographic or clinical characteristics surveyed in this study. 
Table 1 Demographic and clinical characteristics of the participants

\begin{tabular}{|c|c|c|c|}
\hline & \\
\hline Variable & Frequency (\%) N (167) & Variable & Frequency (\%) N (167) \\
\hline Age category (year) & & $<4$ & $87(52.1)$ \\
\hline$<60$ & $80(47.9)$ & $\geq 4$ & $80(47.9)$ \\
\hline$\geq 60$ & $87(52.1)$ & Transplantation history & \\
\hline Gender & & Yes & $16(9.6)$ \\
\hline Male & $87(52.1)$ & No & $151(90.4)$ \\
\hline Female & $80(47.9)$ & Total chronic co-morbid diseases & \\
\hline BMI & & None & $14(8.4)$ \\
\hline Underweight & $5(3.0)$ & 1 & $38(22.8)$ \\
\hline Normal & $59(35.3)$ & 2 & $61(36.5)$ \\
\hline Overweight & $51(30.5)$ & $\geq 3$ & $54(32.4)$ \\
\hline Obese & $52(31.1)$ & Chronic medications & \\
\hline Education & & $<4$ & $64(38.3)$ \\
\hline No formal education & $10(6.0)$ & $\geq 4$ & $103(61.7)$ \\
\hline
\end{tabular}

Primary

$62(37.1)$

Secondary

$32(19.2)$

High

Graduated

$32(19.2)$

31 (18.6)

Household income (month)

High (more than 5000 NIS)

$5(3.0)$

Moderate (2000-4999 NIS)

$51(30.5)$

Low (less than 2000 NIS)

$111(66.5)$

Residency

Refugee camps

$27(16.2)$

Village

Town

$67(40.1)$

$73(43.7)$

Living status

Alone

$10(6.0)$

With family

$157(94.0)$

Marital status

Single, divorced, widowed

36 (21.6)

Married

$131(78.4)$

Occupation

Employed

$20(12.0)$

Unemployed

$127(76.0)$

House wife

$20(12.0)$

Dialysis vintage (years)

$<4$

$99(59.3)$

$\geq 4$

$68(40.7)$

Dialysis per week

$\leq 2$

5 (3.0)

$158(94.6)$

3

$\geq 4$

$4(2.4)$
Table 1 Demographic and clinical characteristics of the participants (Continued)

The results of this study regarding the poor quality among HD patients are in concordance with the results of many similar studies that were conducted to assess the sleep quality in these patients. Such studies have been conducted for many years now and new studies are still emerging with more or less consistent findings regarding the prevalence of this problem. For example, one study using data collected between the years 1996 and 2001 and included 11,351 participants from 308 facilities across 7 countries (France, Germany, Italy, Japan, Spain, the United Kingdom, and the United States), found that almost half the patients (49\%) experienced poor sleep quality and that those who experienced poor sleep quality had a higher risk of mortality (Elder et al. 2008). Such poor sleep quality is even more prevalent as found in this study, affecting as much as $76.65 \%$ of the participants.

This rise in prevalence indicates that it might be helpful to look into the results of more recent studies investigating this matter. A study from China that has used data collected in 2010 reported a $74.49 \%$ prevalence of poor sleep quality among dialysis patients using the same tool employed in the current study (Lai et al. 2015), which is quite similar to the results of this study. Another study conducted in Turkey in 2014 reported that $63 \%$ of HDP had poor sleep quality, though it used a different tool for sleep quality assessment, which might explain the difference in the exact prevalence of the problem (Tosun et al. 2015). To list one more example, a 2018 Pakistani study that included 113 dialysis patients and employed the same assessment tool as in our current study (PSQI) found that $72.6 \%$ of the subjects had poor sleep quality (Anwar and Mahmud 2018), which is also in agreement with our findings. 
Table 2 Relationship between characteristics of participants and their Epworth sleepiness scale and PSQI Scores

\begin{tabular}{|c|c|c|c|c|}
\hline Variable & $\begin{array}{l}\text { ESS } \\
\text { Median [Q1-Q3] }\end{array}$ & $P$ value & $\begin{array}{l}\text { PSQI } \\
\text { Median [Q1-Q3] }\end{array}$ & $P$ value \\
\hline \multicolumn{5}{|l|}{ Age category (Year) } \\
\hline$<60$ & $6.0[3.3-10.8]$ & $0.076^{b}$ & $8.5[6.0-12.0]$ & $0.351^{b}$ \\
\hline$\geq 60$ & $9.0[4.0-12.0]$ & & $8.0[6.0-11.0]$ & \\
\hline \multicolumn{5}{|l|}{ Gender } \\
\hline Male & $8.0[4.0-11.0]$ & $0.339^{b}$ & $8.0[5.0-11.0]$ & $0.113^{b}$ \\
\hline Female & $6.5[3.3-11.0]$ & & $9.0[6.0-12.8]$ & \\
\hline \multicolumn{5}{|l|}{ BMl } \\
\hline Underweight & $14.0[9.5-15.0]$ & & $10.0[8.0-15.5]$ & \\
\hline Normal & $7.0[4.0-11.0]$ & $0.100^{c}$ & $8.0[6.0-11.0]$ & $0.422^{c}$ \\
\hline Overweight & $8.0[4.0-12.0]$ & & $9.0[5.0-13.0]$ & \\
\hline Obese & $7.5[3.3-10.0]$ & & $7.0[5.3-10.8]$ & \\
\hline \multicolumn{5}{|l|}{ Education } \\
\hline No formal education & $5.0[2.0-15.3]$ & & $7.5[5.8-9.8]$ & \\
\hline Primary & $7.0[4.0-11.0]$ & & $9.0[6.0-12.0]$ & \\
\hline Secondary & $8.5[4.5-12.0]$ & $0.459^{c}$ & $9.0[6.0-13.8]$ & $0.475^{c}$ \\
\hline High & $9.5[4.0-11.0]$ & & $8.0[4.0-11.0]$ & \\
\hline Graduated & $6.0[3.0-10.0]$ & & $8.0[5.0-11.0]$ & \\
\hline \multicolumn{5}{|l|}{ Household income (month) } \\
\hline High (more than 5000 NIS) & $6.0[2.5-13.5]$ & & $11.0[5.0-12.5]$ & \\
\hline Moderate (2000-4999 NIS) & $6.0[3.0-10.0]$ & $0.375^{c}$ & $8.0[4.0-13.0]$ & $0.894^{c}$ \\
\hline Low (less than 2000 NIS) & $8.0[4.0-11.0]$ & & $8.0[6.0-11.0]$ & \\
\hline \multicolumn{5}{|l|}{ Residency } \\
\hline Refugee camps & $6.0[3.0-12.0]$ & & $9.0[6.0-10.0]$ & \\
\hline Village & $7.0[4.0-11.0]$ & $0.916^{c}$ & $8.0[5.0-12.0]$ & $0.902^{c}$ \\
\hline Town & $8.0[4.0-11.0]$ & & $8.0[6.0-13.0]$ & \\
\hline \multicolumn{5}{|l|}{ Living status } \\
\hline Alone & $4.5[1.8-12.5]$ & $0.534^{b}$ & $9.0[6.8-13.3]$ & $0.363^{b}$ \\
\hline With family & $7.0[4.0-11.0]$ & & $8.0[5.5-12.0]$ & \\
\hline \multicolumn{5}{|l|}{ Marital status } \\
\hline Single, divorced, widowed & $5.0[3.0-10.0]$ & $0.054^{b}$ & $9.0[6.3-11.0]$ & $0.770^{b}$ \\
\hline Married & $8.0[4.0-11.0]$ & & $8.0[5.0-12.0]$ & \\
\hline \multicolumn{5}{|l|}{ Occupation } \\
\hline Employed & $7.0[4.0-11.0]$ & & $8.0[6.0-11.0]$ & \\
\hline Unemployed & $6.0[3.0-10.8]$ & $0.161^{c}$ & $8.0[4.3-12.8]$ & $0.580^{c}$ \\
\hline House wife & $9.5[6.0-11.8]$ & & $9.0[6.0-14.8]$ & \\
\hline \multicolumn{5}{|l|}{ Dialysis vintage (years) } \\
\hline$<4$ & $6.0[3.0-11.0]$ & $0.236^{b}$ & $9.0[5.0-12.0]$ & $0.670^{b}$ \\
\hline$\geq 4$ & $8.0[4.0-12.0]$ & & $8.0[6.0-11.8]$ & \\
\hline \multicolumn{5}{|l|}{ Dialysis per week } \\
\hline$\leq 2$ & $9.0[5.0-9.5]$ & $0.931^{c}$ & $5.0[3.5-12.5]$ & $0.393^{c}$ \\
\hline 3 & $7.0[4.0-11.0]$ & & $8.5[6.0-12.0]$ & \\
\hline$\geq 4$ & $8.5[8.0-9.8]$ & & $5.0[3.3-8.3]$ & \\
\hline
\end{tabular}

Dialysis session duration (hours) 
Table 2 Relationship between characteristics of participants and their Epworth sleepiness scale and PSQI scores (Continued)

\begin{tabular}{|c|c|c|c|c|}
\hline Variable & $\begin{array}{l}\text { ESS } \\
\text { Median [Q1-Q3] }\end{array}$ & $P$ value & $\begin{array}{l}\text { PSQI } \\
\text { Median [Q1-Q3] }\end{array}$ & $P$ value \\
\hline$<4$ & $6.0[4.0-11.0]$ & $0.443^{b}$ & $8.0[6.0-12.0]$ & $0.987^{b}$ \\
\hline$\geq 4$ & $8.0[4.0-12.0]$ & & $8.0[5.0-12.0]$ & \\
\hline \multicolumn{5}{|c|}{ Transplantation history } \\
\hline Yes & $6.5[3.0-11.8]$ & $0.813^{b}$ & $8.5[5.5-11.0]$ & $0.827^{b}$ \\
\hline No & $7.0[4.0-11.0]$ & & $8.0[6.0-12.0]$ & \\
\hline \multicolumn{5}{|c|}{ Total chronic co-morbid diseases ${ }^{\mathrm{a}}$} \\
\hline None & $6.0[1.0-11.0]$ & & $8.5[7.5-11.3]$ & \\
\hline 1 & $7.0[3.0-10.3]$ & $0.570^{c}$ & $8.0[5.0-11.0]$ & $0.759^{c}$ \\
\hline 2 & $8.0[4.0-12.0]$ & & $9.0[6.0-12.0]$ & \\
\hline$\geq 3$ & $8.0[4.0-11.5]$ & & $7.5[6.0-13.0]$ & \\
\hline \multicolumn{5}{|c|}{ Chronic medications } \\
\hline$<4$ & $7.0[4.0-11.0]$ & $0.756^{b}$ & $9.0[6.0-12.0]$ & $0.166^{b}$ \\
\hline$\geq 4$ & $7.0[4.0-11.0]$ & & 8.0 [6.0-12.0] & \\
\hline
\end{tabular}

Abbreviations: PSQI Pittsburgh Sleep Quality Index, ESS Epworth Sleepiness Scale, NIS New Israeli Shekel, BMI Body Mass Index

${ }^{a}$ Chronic co-morbid diseases include diabetes mellitus, hypertension, chronic rheumatologic disorders, previous myocardial infarction or stroke, heart failure,

chronic lung disease, thyroid disorders, osteoporosis

${ }^{b}$ Statistical significance of differences calculated using the Mann-Whitney $U$ test

' Statistical significance of differences calculated using the Kruskal-Wallis test

Regarding the participants' characteristics that we have focused on, to correlate their relation to sleep quality in these participants, our results are in agreement with some previous studies in some aspectrs, but differ in others. For example, one 2018 randomized trial concluded that no significant association was found between extended hours dialysis and an improvement in sleep quality among 200 dialysis patients (Liao et al. 2019). This parallels our results on the association between the number and duration of dialysis sessions and the participants' sleep quality (both the number and duration of the sessions were not significantly associated with sleep quality). However, this same trial (Liao et al. 2019) found that women had poorer sleep quality than men whereas the association between the participants' gender and their sleep quality was found to be insignificant in our study. Such difference in the findings of the two studies can be the result of some social or environmental factors between the participants of each study, but we cannot draw a final conclusion as to what is causing this difference from the available data.

\section{Strengths and limitations}

To the best of our knowledge, this is the first study to investigate the relationship between regular HD and sleep quality and related problems in Palestine by using two common scales for sleep assessment (i.e. PSQI, and ESS). This study has some limitations. First, its sample was restricted to only one HD centre and not representative to all HD centres in Palestine. Second, because we used a cross-sectional design, strict cause-effect interpretations are not possible. Finally, the results may be biased, due to the convenience sampling methods.

\section{Conclusions}

In conclusion, we found that HD patients in this study had poor sleep quality, evident by the high PSQI score that significantly exceeded the upper limit of normal PSQI score, though their excessive daytime sleepiness was less evident, with a median ESS score in the high normal values. Additionally, the prevalence of poor sleep quality (PSQI $>5$ ) among HD patients in this study was $76.65 \%$, whereas the prevalence of excessive daytime sleepiness (ESS > 10) was $28.7 \%$. Finally, we found no significant association between either of these two scores and the participants' demographic or clinical characteristics surveyed in this study.

Based on our findings, we recommend further investigation of the contributing, as well as the associated factors regarding dialysis patients' sleep quality, such as dialysis shifts timing, sleep hygiene, patients' quality of life, depression, and other underlying relevant health problem that we did not address in this study and to expand such investigation to include more dialysis centers in Palestine. We also recommend implementing the appropriate interventions to address the problem of poor sleep quality among dialysis patients. Such intervention may include sleep hygiene education, physical exercise, and cognitive-behavioral therapy, among other pharmacological and non-pharmacological modalities. 


\section{Abbreviations}

BMI: Body Mass Index; CKD: Chronic kidney disease; DM: Diabetes mellitus; ESS: Epworth Sleepiness Scale; HD: Hemodialysis; HDP: Hemodialysis patients; IRB: Institutional Review Board; NIS: New Israeli Shekel; PSQI: Pittsburgh Sleep Quality Index; RRT: Renal replacement therapy; SPSS: Statistical Package for Social Sciences

\section{Acknowledgments}

The authors thank retired British Library curator Andy Simons for English editing of the manuscript. Also, the authors wish to thank An-Najah National University and An-Najah National University Hospital for their part in making this study possible.

\section{Authors' contributions}

$\mathrm{AO}, \mathrm{LO}$ and HR collected data, performed the analyses, searched the literature and drafted the manuscript. AS and MS performed the analyses, searched the literature, participated in the conception, design, data interpretation and drafted the manuscript. WS and NN participated in the conception, design, data interpretation and manuscript revision. SZ conceptualised and designed the study; coordinated, supervised and took responsibility for the integrity of the data and the accuracy of the data analysis; critically reviewed the manuscript and the interpretation of the results, and assisted in the final write-up of the manuscript. All authors read and approved the final manuscript.

\section{Funding}

No financial funding was received for the current study. No funding of any type was received for developing this survey, collecting or analyzing the data, writing or submitting the manuscript.

\section{Availability of data and materials}

The raw data on which the results in the current study are based may be requested from the corresponding author upon reasonable request.

\section{Ethics approval and consent to participate}

This study was approved by the Institutional Review Board (IRB) at An-Najah National University before starting the study. Also, the appropriate informed consents were obtained from the study's participants before proceeding with the interviews.

\section{Consent for publication}

Not applicable.

\section{Competing interests}

The authors declare that they have no competing interests.

\section{Author details}

'Department of Medicine, College of Medicine and Health Sciences, An-Najah National University, Nablus 44839, Palestine. ${ }^{2}$ Department of Physiology, Pharmacology, and Toxicology, College of Medicine and Health Sciences, An-Najah National University, Nablus 44839, Palestine. ${ }^{3}$ Public Health Department, College of Medicine and Health Sciences, An-Najah National University Hospital, An-Najah National University, Nablus 44839, Palestine. ${ }^{4}$ Poison Control and Drug Information Center (PCDIC), College of Medicine and Health Sciences, An-Najah National University, Nablus 44839, Palestine. ${ }^{5}$ Department of Clinical and Community Pharmacy, College of Medicine and Health Sciences, An-Najah National University, Nablus 44839, Palestine. ${ }^{6}$ Clinical Research Center, An-Najah National University Hospital, Nablus 44839, Palestine.

Received: 30 May 2019 Accepted: 12 August 2019

Published online: 20 August 2019

\section{References}

Abecassis M, Bartlett ST, Collins AJ, Davis CL, Delmonico FL, Friedewald JJ, Hays R, Howard $\mathrm{A}$, Jones $\mathrm{E}$, Leichtman $\mathrm{AB}$. Kidney transplantation as primary therapy for end-stage renal disease: a National Kidney Foundation/kidney disease outcomes quality initiative (NKF/KDOQI ${ }^{\mathrm{m}}$ ) conference. Clin J Am Soc Nephrol. 2008;3(2):471-80
Adas H, Al-Ramahi R, Jaradat N, Badran R. Assessment of adequacy of hemodialysis dose at a Palestinian hospital. Saudi J Kidney Dis Transpl. 2014; 25(2):438-42.

Ahmed AE, Fatani A, Al-Harbi A, Al-Shimemeri A, Ali YZ, Baharoon S, Al-Jahdali H. Validation of the Arabic version of the Epworth sleepiness scale. J Epidemiol Glob Health. 2014:4(4):297-302.

Al-Jabi SW, Sous A, Jorf F, Taqatqa M, Allan M, Sawalha L, Lubadeh E, Zyoud SH, Sweileh WM. Depression in patients treated with haemodialysis: a crosssectional study. Lancet. 2018;391(Suppl 2):S41.

Al-Ramahi R, Raddad AR, Rashed AO, Bsharat A, Abu-Ghazaleh D, Yasin E, Shehab O. Evaluation of potential drug- drug interactions among Palestinian hemodialysis patients. BMC Nephrol. 2016;17:96.

Anwar N, Mahmud SN. Quality of sleep in CKD patients on chronic hemodialysis and the effect of Dialysis shift. J Coll Physicians Surg Pak. 2018;28(8):636-9.

Beaudreau SA, Spira AP, Stewart A, Kezirian EJ, Lui LY, Ensrud K, Redline S, AncoliIsrael S, Stone KL, Study of Osteoporotic F. Validation of the Pittsburgh sleep quality index and the Epworth sleepiness scale in older black and white women. Sleep Med. 2012;13(1):36-42.

Buysse DJ, Reynolds CF 3rd, Monk TH, Berman SR, Kupfer DJ. The Pittsburgh sleep quality index: a new instrument for psychiatric practice and research. Psychiatry Res. 1989;28(2):193-213.

Elder SJ, Pisoni RL, Akizawa T, Fissell R, Andreucci VE, Fukuhara S, Kurokawa K, Rayner HC, Furniss AL, Port FK, et al. Sleep quality predicts quality of life and mortality risk in haemodialysis patients: results from the Dialysis outcomes and practice patterns study (DOPPS). Nephrol Dial Transplant. 2008;23(3):998-1004

Johns MW. A new method for measuring daytime sleepiness: the Epworth sleepiness scale. Sleep. 1991;14(6):540-5.

Kaza BNK, Sabi KA, Amekoudi EYM, Imangue G, Al-Torayhi MH, Tsevi CM, Zamd M, Medkouri G, Benghanem MG, Ramdani B. Kidney transplantation: hemodialysis and surrounding are they informed enough? Indian J Transplant. 2015:9(3):101-4.

Khatib ST, Hemadneh MK, Hasan SA, Khazneh E, Zyoud SH. Quality of life in hemodialysis diabetic patients: a multicenter cross-sectional study from Palestine. BMC Nephrol. 2018;19(1):49.

Kidney Disease: Improving Global Outcomes (KDIGO) CKD Work Group. KDIGO 2012 clinical practice guideline for the evaluation and management of chronic kidney disease. Kidney Int Suppl. 2013:3:1-150.

Lai X, Chen W, Bian X, Wang T, Li J, Wang H, Guo Z. Predictors of poor sleep quality and excessive daytime sleepiness in peritoneal dialysis patients. Ren Fail. 2015;37(1):61-5.

Liao JL, van den Broek-Best O, Smyth B, Hong D, Vo K, Zuo L, Gray NA, Chan CT, de Zoysa J, Perkovic $V$, et al. The effect of extended hours dialysis on sleep quality in a randomised trial. Nephrology (Carlton). 2019;24(4):430-37.

Ministry of Health, Palestinian Health Information Center. Health Status, Palestine, 2015. 2016. http://www.moh.ps/Content/Books/NWNJXX7RJ92Bn4f5EGYiH43 a2tjAAzKBnseGnEUCaqWqYZndsbCcPy_JQWguvkHTR4Xk4zUpdT45ooWxH11 BhlbVAxwpGWy2wiwHdGcM5K7aZ.pdf (Accessed 17 Feb 2017).

Naalweh KS, Barakat MA, Sweileh MW, Al-Jabi SW, Sweileh WM, Zyoud SH. Treatment adherence and perception in patients on maintenance hemodialysis: a cross - sectional study from Palestine. BMC Nephrol. 2017; 18(1):178.

Omari AM, Omari LS, Dagash HH, Sweileh WM, Natour N, Zyoud SH. Assessment of nutritional status in the maintenance of haemodialysis patients: a crosssectional study from Palestine. BMC Nephrol. 2019;20(1):92.

Suleiman KH, Yates BC, Berger AM, Pozehl B, Meza J. Translating the Pittsburgh sleep quality index into Arabic. West J Nurs Res. 2010;32(2):250-68.

Tosun N, Kalender N, Cinar Fl, Bagcivan G, Yenicesu M, Dikici D, Kaya D. Relationship between dialysis adequacy and sleep quality in haemodialysis patients. J Clin Nurs. 2015;24(19-20):2936-44.

Wang R, Tang C, Chen X, Zhu C, Feng W, Li P, Lu C. Poor sleep and reduced quality of life were associated with symptom distress in patients receiving maintenance hemodialysis. Health Qual Life Outcomes. 2016;14(1):125.

World Health Organization. Shortages of supplies for health services, West Bank and Gaza. 2012. http://www.emro.who.int/images/stories/palestine/documents/ pdf/5_Drug_Shortges_WBG_March_2012.pdf (Accessed 2 Feb 2018).

Younis M, Jabr S, Al-Khatib A, Forgione D, Hartmann M, Kisa A. A cost analysis of kidney replacement therapy options in Palestine. Inquiry. 2015;52. https://doi. org/10.1177/0046958015573494

Zyoud SH, Al-Jabi SW, Bali Yl, Al-Sayed AM, Sweileh WM, Awang R. Availability of treatment resources for the management of acute toxic exposures and 
poisonings in emergency departments among various types of hospitals in Palestine: a cross-sectional study. Scand J Trauma Resusc Emerg Med. 2014; 22:13.

Zyoud SH, Al-Jabi SW, Sweileh WM, Tabeeb GH, Ayaseh NA, Sawafta MN, Khdeir RL, Mezyed DO, Daraghmeh DN, Awang R. Use of complementary and alternative medicines in haemodialysis patients: a cross-sectional study from Palestine. BMC Complement Altern Med. 2016a;16:204.

Zyoud SH, Daraghmeh DN, Mezyed DO, Khdeir RL, Sawafta MN, Ayaseh NA, Tabeeb GH, Sweileh WM, Awang R, Al-Jabi SW. Factors affecting quality of life in patients on haemodialysis: a cross-sectional study from Palestine. BMC Nephrol. 2016b;17(1):44.

\section{Publisher's Note}

Springer Nature remains neutral with regard to jurisdictional claims in published maps and institutional affiliations.

Ready to submit your research? Choose BMC and benefit from:

- fast, convenient online submission

- thorough peer review by experienced researchers in your field

- rapid publication on acceptance

- support for research data, including large and complex data types

- gold Open Access which fosters wider collaboration and increased citations

- maximum visibility for your research: over $100 \mathrm{M}$ website views per year

At BMC, research is always in progress.

Learn more biomedcentral.com/submissions 